\title{
A statistical study of the upstream intermediate ion boundary in the Earth's foreshock
}

\author{
K. Meziane*, C. d'Uston \\ Centre d'Etude Spatiale des Rayonnements, 9 Avenue du Colonel Roche, BP 4346, F-31028, Toulouse, Cedex, France
}

Received: 26 January 1996 / Revised: 9 July 1997 / Accepted: 24 July 1997

\begin{abstract}
A statistical investigation of the location of onset of intermediate and gyrating ion populations in the Earth's foreshock is presented based on Fixed Voltage Analyzer data from ISEE 1. This study reveals the existence of a spatial boundary for intermediate and gyrating ion populations that coincides with the reported ULF wave boundary. This boundary position in the Earth's foreshock depends strongly upon the magnetic cone angle $\theta_{B X}$ and appears well defined for relatively large cone angles, though not for small cone angles. As reported in a previous study of the ULF wave boundary, the position of the intermediate-gyrating ion boundary is not compatible with a fixed growth rate of the waves resulting from the interaction between a uniform beam and the ambient plasma. The present work examines the momentum associated with protons which travel along this boundary, and we show that the variation of the boundary position (or equivalently, the associated particle momentum) with the cone angle is related to classical acceleration mechanisms at the bow shock surface. The same functional behavior as a function of the cone angle is obtained for the momentum predicted by an acceleration model and for the particle momentum associated with the boundary. However, the model predicts systematically larger values of the momentum than the observation related values by a constant amount; we suggest that this difference may be due to some momentum exchange between the incident solarwind population and the backstreaming particles through a wave-particle interaction resulting from a beam plasma instability.
\end{abstract}

Key words Intermediate ion boundary $\cdot$ Statistical investigation - Earth's foreshock - ISEE 1 spacecraft

\footnotetext{
* Present address: Geophysics Program, University of Washington, Seattle, WA 98195, USA

Correspondence to: K. Meziane

e-mail: meziane@geophys.washington.edu
}

\section{Introduction}

A wealth of information on the Earth's foreshock collected by the ISEE 1 and 2 spacecraft has been studied extensively for the last two decades. Several types of upstream ion distribution function have been identified (Gosling et al., 1978; Paschmann et al., 1981; Gurgiolo et al., 1981), and extensive observational and theoretical investigations have been undertaken to explain the origin and behavior of these populations. Reflected ion beams and diffuse ion populations represent the two limits of the inferred distribution functions, the former characterized by a narrow angular distribution collimated along interplanetary magnetic field lines, the latter by an almost isotropic pitch-angle distribution. The high-energy $(E>10 \mathrm{keV})$ field-aligned beams are often observed in the vicinity of the ion foreshock edge and are accelerated near the perpendicular shock. Lowenergy $(E \sim 2 \mathrm{keV})$ beams, observed in the quasiperpendicular region, are believed to be produced by an adiabatic reflection of a portion of incoming solarwind ions (Paschmann et al., 1980) or also by leakage from the magnetosheath (Thomsen et al., 1983a). Intermediate ion distributions, which have a crescent shape in velocity space centered around the magnetic field direction, have also been identified. High-timeresolution analysis has shown that many intermediate ion distributions in fact have the signature of gyrating ions. These gyrating ions, in which the distribution peaks at nonzero pitch-angles, may be gyrotropic or phase-bunched (Thomsen et al., 1985; Fuselier et al., 1986).

Intermediate, gyrating, and diffuse ion populations are often observed simultaneously with low-frequency MHD waves with amplitudes that may reach a large fraction of the ambient magnetic field magnitude (Paschmann et al., 1979; Hoppe et al., 1981). The waves associated with gyrating and intermediate ion populations are quasi- monochromatic, right-handed in the 
plasma rest frame, and propagate nearly parallel to the ambient magnetic field, while those observed with diffuse ions are more steepened. Several models involving the ion beam instability resulting from the interaction between the reflected ion population and the solar-wind ion distribution have been developed to explain the simultaneous occurrence of low-frequency waves and gyrating, intermediate, and diffuse ions (Gary et al., 1981; Lee, 1982; Winske and Leroy, 1984). In these models, the propagation of field-aligned beams through the solar-wind plasma excites electromagnetic waves through the ion-beam instability. The excited waves may trap ions early in the wave-growth process producing gyrophase-bunched populations observed within several Earth radii from the shock (Hoshino and Teresawa, 1985). Alternatively, the excited waves may also induce pitch-angle scattering leading to intermediate distributions (Fuselier, 1995). In both cases the field-aligned beams are destroyed. Isotropization during the nonlinear part of the wave-particle interaction ultimately produces a diffuse ion population. Thus, gyrating, intermediate, and diffuse ion distributions may all result from the disruption of field-aligned distributions through pitch-angle scattering by MHD waves generated by the ion-beam instability. If these models are assumed, the growth rate of the instability allows use to infer the region within the foreshock where the ion beam-solar wind interaction occurs (Lee and Skadron, 1985).

Using the AMPTE/IRM diffuse ion measurements, Möbius et al. (1987) and Trattner et al. (1994) have shown that the wave energy density measured in association with diffuse ion events agrees well with the self-consistent model of Lee (1982). The propagation of upstream diffuse ion populations in the quasi-parallel shock region can also excite low-frequency MHD waves, because their mean energy is larger than the solar-wind mean energy. However, in this case the growth rate is smaller than that obtained in the beam plasma instability (Sentman et al., 1981) and remains too low to explain the observed wave amplitudes (Hada et al., 1987).

Another source of intermediate, gyrating, and diffuse ion populations may be the acceleration by the shock. Ions may leave the shock with a gyrophase organization (Gurgiolo et al., 1983), and these bunched ions can undergo further gyrophase mixing within a few Earth radii from the shock. Alternatively, gyrotropic ions (ring-beam) may be accelerated in the quasi-parallel shock (Gosling et al., 1982). Direct emission of intermediate ions through escape from the magnetosheath has been demonstrated theoretically (Edmiston et al., 1982). Acceleration by the shock is also probably an important source of diffuse ions. Most evidence for the validity of this hypothesis comes from upstream ion events observed during nearly radial IMF (Möbius et al., 1987; Trattner et al., 1994). When the IMF direction is quasi-radial, the field-aligned beam accelerated in the perpendicular shock cannot propagate deeply downstream in the quasi-parallel region where diffuse ions are observed. This may be explained by solar-wind ions that suffer several reflections between the magnetic turbulence in the quasi-parallel region and the shock itself to produce the suprathermal diffuse ion distribution (Lee, 1982). Other evidence that the field-aligned beam is not the only source of diffuse ions concerns the composition measurement of upstream ions. Diffuse ions contain substantial numbers of $\mathrm{He}^{2+}$ particles (at approximately the solar-wind fraction), whereas the field-aligned beam composition has very little $\mathrm{He}^{2+}$ (Ipavich et al., 1984, 1988; Fuselier and Thomsen, 1992), which suggests that the source of diffuse ions is the solar wind rather the magnetosphere (Fuselier, 1994). Hybrid simulations of quasi-parallel collisionless shocks (Quest, 1988; Scholer and Teresawa, 1990) show that some incoming ions undergo multiple reflection at the quasiparallel shock and are accelerated to higher energies, escaping upstream with a velocity distribution function similar to a diffuse ion distribution (Scholer, 1990).

Through a statistical survey of ISEE 1 magnetometer data, Greenstadt and Baum (1986) demonstrated the existence of a boundary for the ultra-low-frequency (ULF) compressional wave region. The ULF wave foreshock boundary was identified when the spacecraft passed from a region where no IMF fluctuations were detected into a region where ULF waves were observed, or vice versa. This ULF wave foreshock boundary is characterized by a sudden variation of all three components of the IMF while the mean orientation holds steady (no important variation of IMF direction), providing a signature which enables a good determination of its location. Greenstadt and Baum did not discuss the characteristics of the waves associated with the ULF boundary, particularly whether these waves have large amplitudes, are monochromatic or are steepened, and made no distinction between the types of ULF waves. In the $\vec{B}-\vec{V}_{s w}$ plane, their boundary appears as a straight line which is not parallel to the average trajectory of field-aligned beams and has a position that depends strongly on the so-called cone angle $\theta_{B X}$ (the angle between the IMF and the SunEarth direction). Thus, Greenstadt and Baum (1986) rejected any model of wave production by beam-solar wind interaction that depends on uniform beam distribution or fixed growth rate. Instead, their findings supported the idea of a separate escaping population from the shock surface itself.

More recently, Le and Russell (1992) selected 373 bow shock crossings at various geometries and found a precise limit of ULF wave foreshock boundary where IMF fluctuations fill a foreshock region corresponding to $\theta_{B n} \leq 50^{\circ}$, where $\theta_{B n}$ is the angle between the IMF and the bow shock normal; no ULF waves are observed for higher $\theta_{B n}$ values.

The association of ULF waves and upstream ions in the foreshock leads us to expect the existence of a spatial ion foreshock boundary similar to the reported ULF wave boundary. This paper demonstrates its existence, for which no direct evidence of this boundary has previously been reported. The observation and the characterization of this boundary is an essential way of testing the validity of models involving wave and particle interactions. The method of data analysis is 
described in the next section. Section 3 presents results based on a survey of the ion measurements from ISEE 1 during November 1977 which are then compared to lowfrequency wave analysis. Finally, the relationship between simple acceleration models of particles at the shock surface and the geometrical characteristics of the boundary will be discussed.

\section{Data selection and method of analysis}

This study is based on the upstream proton fluxes measured by ISSE 1 with the Berkeley-Seattle-Toulouse experiment (Anderson et al., 1978) at fixed energies $(1.6 \pm 0.2 \mathrm{keV}$ and $6.0 \pm 0.6 \mathrm{keV})$. The axis of the detector's field of view is directed roughly normal to the ecliptic plane along the spin axis of the spacecraft. As the interplanetary magnetic field usually lies close to ecliptic plane, these detectors mostly sample particles with large pitch angles and thus detect intermediate, gyrating, or diffuse ions. Some events exhibit rapid flux variations at high time resolution and are often associated with nearly monochromatic MHD waves (Potter, 1985); it is probable that these ions are associated with intermediate or gyrating ion populations (Thomsen et al., 1985; Fuselier et al., 1986). Figure 1 shows an example in front of the bow shock in the interplanetary medium on 2 November 1977 between 00:00 and 12:00 UT, where the $6-\mathrm{keV}$ ion fluxes averaged over $64 \mathrm{~s}$ are presented as a function of time. Fluxes at the background level indicate that the spacecraft is outside the foreshock or in a field-aligned beam region (d'Uston et al., 1986) and they typically appear or disappear abruptly, clearly showing when the spacecraft enters or leaves a spatial ion foreshock boundary.

To locate this ion boundary, we first adopt a measurable criterion of the boundary crossing, set at an intermediate flux level between the background level in the interplanetary medium of $\sim 800$ $\left(\mathrm{cm}^{2} \cdot \mathrm{sr} \cdot \mathrm{keV} \cdot \mathrm{s}\right)^{-1}$ and the higher level of ion flux events, which for $6-\mathrm{keV}$ ions ranges from $\sim 10^{3}$ $\left(\mathrm{cm}^{2} \cdot \mathrm{sr} \cdot \mathrm{keV} \cdot \mathrm{s}\right)^{-1}$ to $\sim 10^{5}\left(\mathrm{~cm}^{2} \cdot \mathrm{sr} \cdot \mathrm{keV} \cdot \mathrm{s}\right)^{-1}$. Figure 2 shows boundary crossing examples as indicated by arrows. At 10:29 UT, the 6-keV flux level abruptly increases from the level in the interplanetary medium by

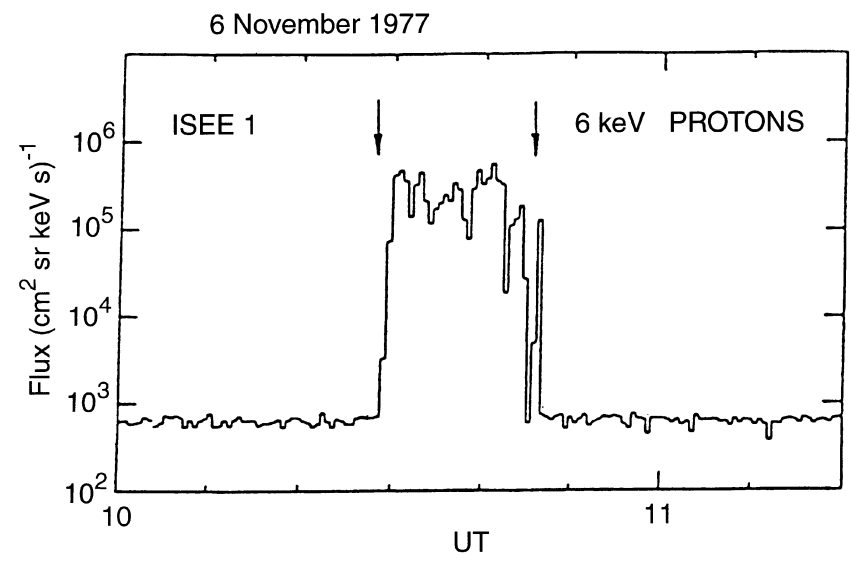

Fig. 2. Intermediate ion boundary crossings (indicated by arrows) inferred from the 6-keV ion flux registered on 6 November 1977

almost three orders of magnitude. The ISEE 1 spacecraft remained inside the foreshock region until 10:45 UT, when it again crossed the boundary.

The foreshock region has a structured topology (Bonifazi and Moreno, 1981), with field-aligned beams present in the quasi-perpendicular region, intermediate ions detected downstream from there, and diffuse ions present even further downstream. Gyrating ions tend to be located in the intermediate ion region, with both populations having the same occurrence probability (Fuselier et al., 1986). As the uni-directional detector cannot discern the nature of the distribution, we therefore assume in the present study that ion events that appear in the detector may be either intermediate or gyrating. We call the ion boundary investigated here the "intermediate ion boundary", knowing that we may also include gyrating ions. Boundary crossings occur because of temporal variations of the interplanetary magnetic field direction as well as spacecraft motion. Geometric parameters that describe the location of the boundary crossing are then computed. The magnetic field direction is taken from the 64-s-averaged values contained in the datapool tapes as measured by the UCLA magnetometer.

To compare the position of these crossings with the boundary of the ULF wave foreshock, we adopt the Solar Foreshock Coordinates (SFC) frame used by Greenstadt and Baum (1986). Figure 3a shows a cross

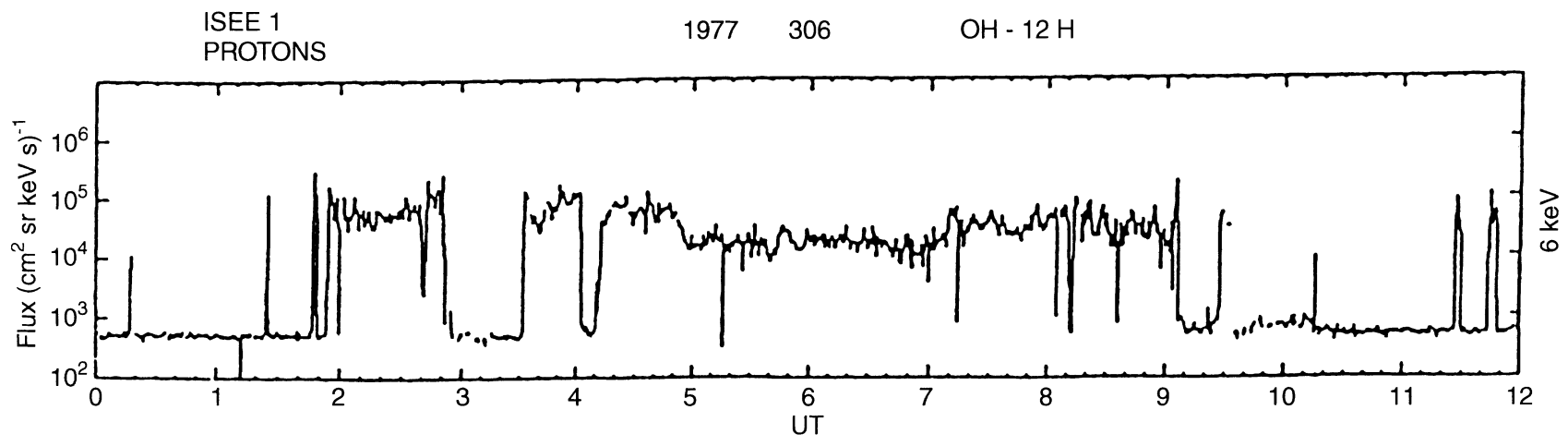

Fig. 1. The 6-keV ion flux registered aboard ISEE 1 on 2 November 1977 between 00:00 and 12:00 UT 

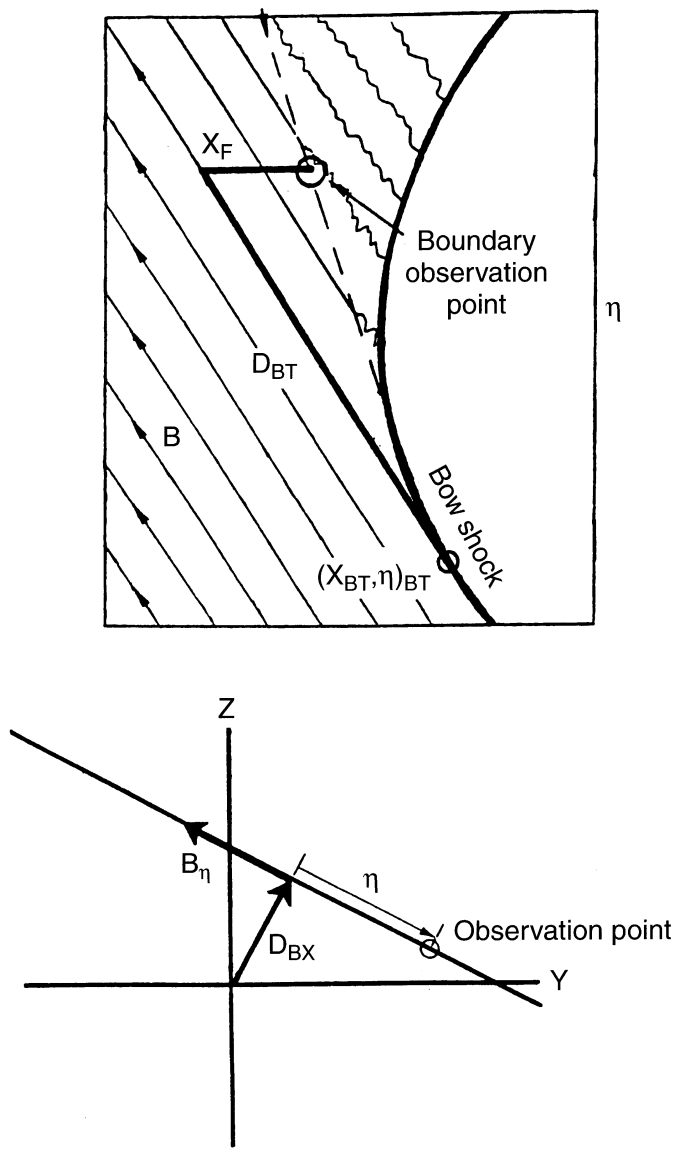

Fig. 3. The SFC system (from Greenstadt and Baum, 1986). The $(X, \eta)$ plane contains the spacecraft location, the solar-wind flow and the interplanetary magnetic field directions. See text for the definition of $X_{F}, D_{B T}$, and $D_{B X}$

section of the bow shock in the $\vec{B}-\vec{V}_{s w}$ plane, defined by the observation point, the interplanetary magnetic field direction and the Sun-Earth axis ( $X$-axis). Backstreaming ions move in the $\vec{B}-\vec{V}_{s w}$ plane and are located by coordinates $X$ and $\eta$ as shown in Fig. 3a. If it is assumed that the bow shock shape is best represented by a hyperboloid, then its cross section in the $(X, \eta)$ plane is described by the equation (Greenstadt and Baum, 1986):
$\eta^{2}=A\left[\left(X-B D_{0}\right)^{2}+C D_{0}^{2}\right]-D_{B X}^{2}$

$A, B$, and $C$ are $0.04,39.22$, and 1461, respectively, constant values chosen to model the hyperboloid shape of the shock; $D_{0}$ is the geocentric distance of the subpolar point (we have adopted $13.5 \mathrm{R}_{\mathrm{E}}$ ) and $D_{B X}$ is the distance between the Sun- Earth axis and the $(X, \eta)$ plane (Fig. 3b). Following Greenstadt and Baum (1986), $X_{F}$ and $D_{B T}$ will be used to locate the observations. $X_{F}$ is the distance from the observation point to the field line tangent to the shock, along a line parallel to the $X$-axis. $D_{B T}$ is the distance along this tangent field line from the shock to the point of the same value of $\eta$ in the $(X, \eta)$ plane (Fig. 3a).

\section{Results}

We have selected 404 events observed at ISEE 1 during November 1977 from an analysis of 6-keV ion fluxes and magnetic field. To study the sensitivity of the intermediate ion boundary to the cone angle, the data have been sorted by $\theta_{B X}$, the angle that the $X$-axis makes with the interplanetary magnetic field direction, and binned according to the value of $\theta_{B X}: 20+10 j \mathrm{deg}$ $<\theta_{B X}<30+10 j \mathrm{deg}$, or $\left\langle\theta_{B X}\right\rangle=25+10 j \mathrm{deg}$, where $j=0,1, \ldots, 6$. The number of events is $140,77,64,65$, 38,12 , and 8 , for $\left\langle\theta_{B X}\right\rangle=25^{\circ}, 35^{\circ}, 45^{\circ}, 55^{\circ}, 65^{\circ}, 75^{\circ}$, and $85^{\circ}$, respectively. The frequency of events decreases when $\theta_{B X}$ increases due to a combination of the spacecraft orbit and the averaged configuration of the interplanetary magnetic field.

The results of the statistical study are illustrated in Fig. 4 for three values of $\left\langle\theta_{B X}\right\rangle: 25^{\circ}$ (Fig. $4 \mathrm{a}$ ), $35^{\circ}$ (Fig. 4b), and $45^{\circ}$ (Fig. 4c). The points represent the position of each crossing of the intermediate ion boundary within the specified range of $\theta_{B X}$. The solid line shows the best-fitting linear approximation of the points, $X_{F}=p D_{B T}+q$, while the dashed line corresponds to the ULF wave foreshock boundary of Greenstadt and Baum (1986). The derived values of $p$ and $q$ with the uncertainties obtained and the associated correlation coefficient $R$ are indicated in Table 1 for each

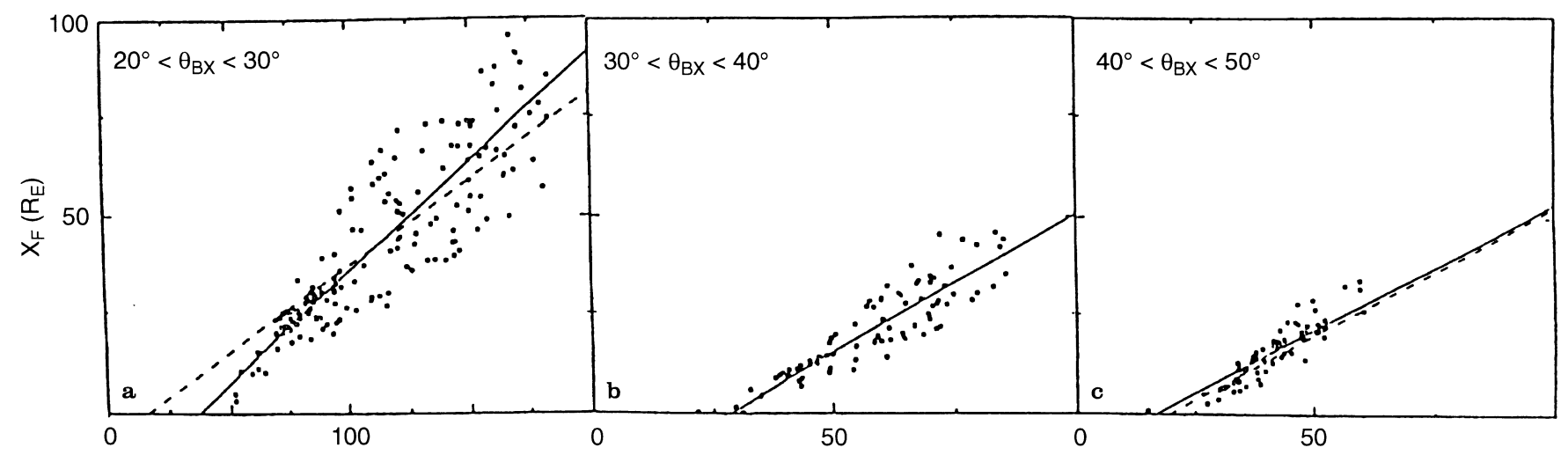

Fig. 4. Scatter plot of the observed intermediate ion boundary crossings in the SFC frame for three $\theta_{B X}$ domains. The straight lines represent the best linear fits to these points. The dashed straight lines represent the ULF wave boundary reported by Greenstadt and Baum (1986) 
Table 1. Coefficients of straight-line boundary of intermediate ions for different $\theta_{B X}$ domains

\begin{tabular}{llllll}
\hline$\theta_{B X}\left({ }^{\circ}\right)$ & $p$ & $q\left(\mathrm{R}_{\mathrm{E}}\right)$ & $R$ & $p_{G B}$ & $q_{G B}\left(\mathrm{R}_{\mathrm{E}}\right)$ \\
\hline $25 \pm 5$ & $0.56 \pm 0.02$ & $-20.60 \pm 2.45$ & 0.91 & 0.44 & -6.97 \\
$35 \pm 5$ & $0.71 \pm 0.03$ & $-20.22 \pm 1.48$ & 0.94 & - & - \\
$45 \pm 5$ & $0.64 \pm 0.02$ & $-10.38 \pm 0.68$ & 0.96 & 0.65 & -12.43 \\
$55 \pm 5$ & $0.66 \pm 0.02$ & $-9.23 \pm 0.61$ & 0.95 & - & - \\
$65 \pm 5$ & $0.67 \pm 0.03$ & $-8.48 \pm 0.64$ & 0.93 & - & - \\
$75 \pm 5$ & $0.61 \pm 0.05$ & $-8.59 \pm 0.81$ & 0.87 & - & - \\
$85 \pm 5$ & $0.58 \pm 0.06$ & $-8.95 \pm 0.93$ & 0.82 & - & - \\
\hline
\end{tabular}

group, as well as $p_{G B}$ and $q_{G B}$, the coefficients associated with the ULF wave foreshock boundary.

Our results are in very good agreement with those obtained by Greenstadt and Baum for $\left\langle\theta_{B X}\right\rangle=45^{\circ}$ (Fig. 4c and Table 1). For this case and $\left\langle\theta_{B X}\right\rangle=35^{\circ}$, the location of the points shows a weak scatter, and the correlation coefficients indicate that the linear fit is good. The excellent agreement of the boundaries of intermediate ion distributions and the MHD lowfrequency waves for moderate cone angles thus verifies in a systematic way, with a relatively large statistical basis, an association previously reported for a limited number of events studied with different instruments (Paschmann et al., 1979; Hoppe et al., 1981; Trattner et al., 1994).

For $\left\langle\theta_{B X}\right\rangle=25^{\circ}$, a significant difference exists between the two boundaries that may be partly due to the relatively larger scatter of our inferred positions. At small $\theta_{B X}$ the IMF lines are tangent to the distant flank of the bow shock, a region where the shock shape model may not be reliable. This uncertainty may produce larger relative scatter of our measurements for $\left\langle\theta_{B X}\right\rangle=25^{\circ}$ and is accentuated by very high values of $D_{B T}$ and $X_{F}\left(\sim 200\right.$ and $\sim 100 \mathrm{R}_{\mathrm{E}}$, respectively $)$. This is in agreement with the ULF wave boundary inferred by Le and Russell (1992), which does not have a welldefined location for small cone angles $\left(\theta_{B X}<30^{\circ}\right)$.

We now examine the particle momentum associated with the ion foreshock boundary. The guiding center velocity, $V_{G C}$, of ions traveling along this boundary may be expressed in terms of a fraction of solar-wind speed, $V_{s w}$ :

$V_{G C}=\sqrt{V_{\|}^{2}+V_{D}^{2}}=P_{i r} V_{S W}$,

where $V_{\|}$and $V_{D}$ are, respectively, the parallel and drift velocity, and $P_{i r}$ is a multiplicative factor indicating the momentum magnitude of the backstreaming ions. Through geometric considerations (see Appendix), $P_{i r}$ can be related to the boundary parameters, allowing us to calculate the corresponding $P_{i r}$ values related to each $\left\langle\theta_{B X}\right\rangle$ domain. For $\left\langle\theta_{B X}\right\rangle=45^{\circ}$, the ion-foreshockboundary-associated $P_{i r}$ is $1.11 \pm 0.04$, in good agreement with the ULF-wave-boundary-associated $P_{i r}$ reported by Greenstadt and Baum $\left(P_{i r}=1.09\right)$. Statistical studies of the bulk flow velocity associated with upstream particles by Bonifazi and Moreno (1981) have found $P_{i r}=2$ for the beam distributions, $P_{i r}=1.75$ for intermediate ion distributions, and $P_{i r}=1.18$ for diffuse ion distributions. The value of $P_{i r}$ found in the present work, however, is not consistent with the average bulk velocity of intermediate ions as determined by Bonifazi and Moreno (1.75) but rather with diffuse ions (1.18). A possible explanation for this discrepancy is that since the detector is only able to detect ions having a relatively large spread of pitch-angle values, the boundary detected here is slightly downstream of the actual intermediate or gyrating ion boundary, i.e., the onset of the diffuse ion region. This also indicates that the intermediate ion foreshock boundary is not parallel to the ion foreshock boundary associated with the beam distribution but may lie closer to the diffuse ion boundary.

The comparison of our $P_{i r}$ values with those obtained by Le and Russell is not straightforward. These authors have located the wave activity region when the spacecraft crosses the bow shock in the upstream region, and consequently they have determined $\theta_{B n}$ values when IMF fluctuations are observed. Identifying only three ULF foreshock boundary crossings for $\left\langle\theta_{B X}\right\rangle=85^{\circ}$, Le and Russell found that the velocity flow associated with the ULF wave foreshock crossings is $P_{i r}=1.9$, in good agreement with our estimate $P_{i r}=1.93 \pm 0.15$. For $\left\langle\theta_{B X}\right\rangle=45^{\circ}$, however, Le and Russell identified five ULF wave foreshock crossings with $P_{i r}=1.5$, significantly higher than our $1.11 \pm 0.04$.

Another interesting aspect of the intermediate ion foreshock boundary concerns its position relative to the shock surface, best studied with $\theta_{B n}$, a parameter sensitive to the shock drift acceleration process. Analysing 373 bow shock crossings, Le and Russell (1992) reported that the ULF foreshock starts at $\theta_{B n}^{*} \approx 50^{\circ}$, where $\theta_{B n}^{*}$ is the angle between the IMF and the bow shock normal projected in the $\vec{B}-\vec{V}_{s w}$ plane. No fluctuations of IMF are seen for $\theta_{B n}^{*} \geq 50^{\circ}$. We note that $\theta_{B n}^{*}$ is always smaller than or equal to $\theta_{B n}$ computed with a three-dimensional bow shock model. Le and Russell used a few crossings of the ULF boundary in the foreshock to define the shape of this boundary and supposed in most cases that it starts at a point on the bow shock with $\theta_{B n}^{*} \leq 50^{\circ}$. They do not discuss whether the ULF foreshock boundary is tangent or not to the bow shock.

The straight-line fits determined in the present work are not strictly tangent to the shock, but we were able to construct tangent lines by relatively small translations in $X$. This is justified by the uncertainties in $p$ and $q$ and the small number of measurements near the shock. With this assumption we calculated $\theta_{B n}$ at the corresponding tangent point. Table 2 lists such computed values of $\theta_{B n}$ and the uncertainties $\Delta \theta_{B n}$ for each cone angle. In agreement with the Le and Russell investigations, the $\theta_{B n}$ values are close to $50^{\circ}$ for $\left\langle\theta_{B X}\right\rangle=35^{\circ}, 45^{\circ}, 55^{\circ}, 65^{\circ}, 75^{\circ}$, and $85^{\circ}$. The $\theta_{B n}$ values obtained by our computation are slightly greater than those of Le and Russell, however, because they are computed with a threedimensional bow shock. In all cases, except for $25^{\circ}$, the values of $\theta_{B n}$ are smaller than $60^{\circ}$, consistent with the fact that very few intermediate and gyrating ion events are observed for $\theta_{B n}$ greater than $60^{\circ}$ (Fuselier et al., 1986). Finally, for $\left\langle\theta_{B X}\right\rangle=25^{\circ}$, the boundary intersects 
Table 2. $\theta_{B n}$ and $\theta_{V n}$ values and their uncertainties for each intermediate ion boundary

\begin{tabular}{lll}
\hline$\theta_{B X}\left({ }^{\circ}\right)$ & $\theta_{B n} \pm \Delta \theta_{B n}\left({ }^{\circ}\right)$ & $\theta_{V n} \pm \Delta \theta_{V n}\left({ }^{\circ}\right)$ \\
\hline $25 \pm 5$ & $65.6 \pm 1.6$ & $42.3 \pm 1.6$ \\
$35 \pm 5$ & $49.7 \pm 2.5$ & $24.3 \pm 1.2$ \\
$45 \pm 5$ & $53.9 \pm 1.4$ & $22.8 \pm 0.2$ \\
$55 \pm 5$ & $52.6 \pm 1.4$ & $23.0 \pm 0.2$ \\
$65 \pm 5$ & $53.1 \pm 2.2$ & $26.2 \pm 0.7$ \\
$75 \pm 5$ & $57.7 \pm 3.6$ & $28.7 \pm 1.8$ \\
$85 \pm 5$ & $60.8 \pm 4.3$ & $32.7 \pm 2.6$ \\
\hline
\end{tabular}

the bow shock at quasi-perpendicular shock geometry; in this case we have no measurements within $100 \mathrm{R}_{\mathrm{E}}$ of the shock. The position of the boundary between 0 and $100 \mathrm{R}_{\mathrm{E}}$ in $D_{B T}$ is obtained by extrapolation of our measurements and hence is subject to larger errors, so the value corresponding $\theta_{B n}$ may not be reliable.

We now compare our calculated boundary with theoretical models of the ULF wave boundary. In the simple plasma-beam instability model of Lee and Skadron (1985), the intermediate ion boundary should be parallel to the beam direction, and no diffuse ion distribution can be formed for $\theta_{B X}>45^{\circ}$. But since the observed ULF wave boundary is not parallel to the beam direction, the observed boundary is incompatible with fixed growth rates and uniform proton beam injection. A model of the formation of the wave compressional foreshock boundary involving a nonuniform beam injection was elaborated by Skadron et al. (1988). In this model, the interaction between the beams and background waves in the solar wind excites outward-propagating, right-hand polarized waves downstream and leads to an upstream boundary for enhanced wave activity. The injected proton beam is characterized by a spatially variable density and a constant velocity in the solar-wind frame. For $\left\langle\theta_{B X}\right\rangle=45^{\circ}$, the model gives a boundary located $78^{\circ}$ away from the Earth-Sun axis, compatible with our computed value of $84.6^{\circ} \pm 7^{\circ}$ and close to the Greenstadt and Baum determination $\left(85^{\circ}\right)$. For $\left\langle\theta_{B X}\right\rangle=25^{\circ}$, the angle of our intermediate ion foreshock boundary relative to the Earth-Sun axis is $50.7^{\circ} \pm 9^{\circ}$, also comparable with the theoretical compressional boundary wave boundary $\left(42^{\circ}\right)$. This good agreement with the theoretical model of Skadron et al. (1988) suggests that, although we do not always agree with the observed ULF boundary of Le and Russell (1992), the theoretical ULF boundary is well correlated with the intermediate ion boundary.

\section{Comparison with emission models}

The foregoing discussion demonstrates the difficulty in explaining the characteristics of the boundary in terms of a fixed growth process arising from instabilities such as the plasma-beam instability. However, an alternative explanation for the properties of the ULF wave foreshock boundary has been reported by Skadron et al. (1988). Their theoretical model assumed an interaction of shock-issued beams with seed interplanetary waves; the emission mechanisms at the shock surface have been ignored. In the following, we examine how the emission processes at the shock can be related to the characteristics of the ion boundary reported in our study. Two physical processes may govern the geometrical properties of the ion foreshock boundary: (1) the emission mechanism at the shock, and (2) the energy exchange between the backstreaming suprathermal ions and the solar wind. The flow speed $\vec{V}_{G C}$ acquired by a particle during a shock emission can be expressed as a function of solar-wind speed $V_{s w}$ by $V_{G C}=P_{i r} V_{s w}$. The expression of $P_{i r}^{2}$ can be expressed as (Schwartz et al., 1983):

$$
\begin{aligned}
P_{i r}^{2}= & 1+\left[\frac{\cos ^{2} \theta_{V n}}{\cos ^{2} \theta_{B n}}\right] f^{2}\left(\theta_{B n}\right) \\
& -\left[\frac{\cos \theta_{V n}}{\cos \theta_{B n}}\right] f\left(\theta_{B n}\right) \cos \theta_{B X},
\end{aligned}
$$

where $\theta_{B n}$ and $\theta_{V n}$ are the angles that the shock normal makes with the magnetic field and the solar-wind direction, respectively, and $f$ is a function of $\theta_{B n}$ which depends on the considered emission process. It is clear that whichever models of emission are considered, Eq. (3) signifies a decreasing linear relation with $\cos \theta_{B X}$.

In Fig. 5 the values of $P_{i r}^{2}$ are reported as a function of $\cos \theta_{B X}$. The associated error bars are evaluated from the uncertainty of the slope $p$, as given in Table 1 . Figure 5 shows that the dependence of $P_{i r}^{2}$ on $\theta_{B X}$ may be approximated well by a linear function of $\cos \theta_{B X}$ (correlation coefficient equal to 0.97), i.e.,

$P_{i r}^{2}=3.8-3.6 \cos \theta_{B X}$.

$P_{i r}^{2}$ represents (when multiplied by $\frac{1}{2} m_{p} V_{s w}^{2}$, where $m_{p}$ is the proton mass) the flow energy of particles traveling along the boundary. As indicated by Eq. (3) and demonstrated in Fig. 5, this energy is strongly dependent on the angle that the IMF makes with the solarwind direction. This indicates that the distribution

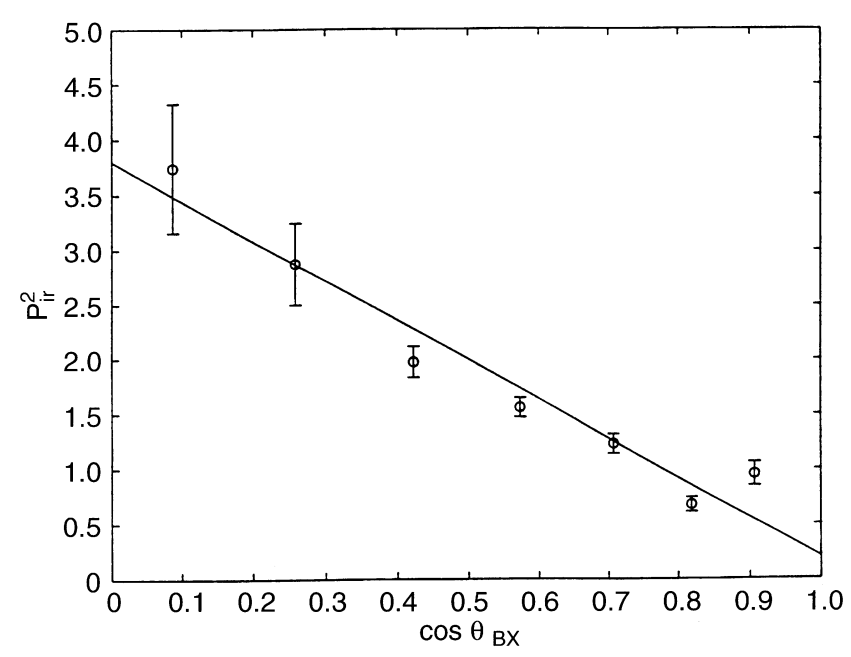

Fig. 5. Diagram showing the dependence of $P_{i r}^{2}$ on $\cos \theta_{B X}$. The straight line corresponds to a best-fit linear approximation 
function of suprathermal ions depends strongly on the angle $\theta_{B X}$ previously reported by Lee and Skadron (1985).

The qualitative agreement between Eq. (3), which expresses the energy gain given by the emission models, and Eq. (4), which expresses the energy flow deduced from measurements and the geometrical characteristics of boundary, leads us to study quantitatively the values of $P_{i r}$ given by Eq. (3). For this, we review briefly four emission models.

1. Adiabatic reflection of solar-wind ions produces an ion beam aligned with the interplanetary magnetic field with generally high energies $\left(P_{i r} \approx 2\right)$ (Thomsen et al., 1983b). In this case, $f=2$.

2. Specular reflection of solar-wind ions gives birth to an emission in the upstream region only for $\theta_{B n}<45^{\circ}$ (Gosling et al., 1982; Schwartz et al., 1983). In this case, $f=2 \cos \theta_{B n}$.

3. Magnetosheath particle leakage with magnetic moment conservation explains particularly the observation of low-energy ion beams $\left(P_{i r}<2\right)$ (Thomsen et al., 1983a). The energy gain depends on the electric potential difference $\Delta \Phi$ across the shock. The maximal potential difference $\Delta \Phi_{\max }$ is $\frac{1}{2} m_{p} V_{s w}^{2}=q \Delta \Phi_{\max }$ (Schwartz et al., 1983), where $q$ is the proton charge. Shock simulations at perpendicular geometries provide values of $\Delta \Phi$ about $60 \%$ of $\Delta \Phi_{\max }$ (Sanderson and Uhrig, 1978; Leroy et al., 1982). In adopting this last value for $\Delta \Phi_{\max }$, we obtain $f=1+0.77 \cos \theta_{B n}$ (Schwartz et al., 1983).

4. Finally, magnetosheath particle leakage occur along the shock normal $\mathbf{n}$. The same previous arguments concerning $\Delta \Phi$ lead to $f=1+0.77 \cos ^{2} \theta_{B n}$.

To compare the different emission models with the observations, we have computed the angles $\theta_{B n}$ and $\theta_{V n}$ for each value of $\left\langle\theta_{B X}\right\rangle$, assuming that the boundaries are tangent to the shock. These quantities are given in Table 2 along with their uncertainties $\Delta \theta_{B n}$ and $\Delta \theta_{V n}$. It appears that the values found for $\theta_{B n}$ and $\theta_{V n}$ are not contaminated with large errors. Consequently, the angles associated with each boundary are reliable enough to permit a comparison of the $P_{i r}$ values given by Eq. (3) with those derived from the observations. In addition, with the exception of $\left\langle\theta_{B X}\right\rangle=25^{\circ}$, the $\theta_{V n}$ values do not deviate considerably from one another as $\left\langle\theta_{B X}\right\rangle$ changes. As the $\theta_{B n}$ values found are all larger than $45^{\circ}$, we can immediately reject the specular reflection hypothesis. Implying that the gyrating ion distributions accelerated at the quasi-parallel shock (Gosling et al., 1982) are probably excluded from our data selection.

For the three other hypotheses, $P_{i r}$ has been calculated and the results are illustrated in Fig. 6, where the experimental and the theoretical values are reported as a function of $\cos \theta_{B X}$. The error bars come from the errors on $\theta_{B n}$ and $\theta_{V n}$. It is apparent that there is a great similarity between the curves, and it is particularly remarkable that the minimal value of $P_{i r}$ obtained experimentally at $\left\langle\theta_{B X}\right\rangle=35^{\circ}$ is well modeled by the minimal value in all emission processes. For adiabatic

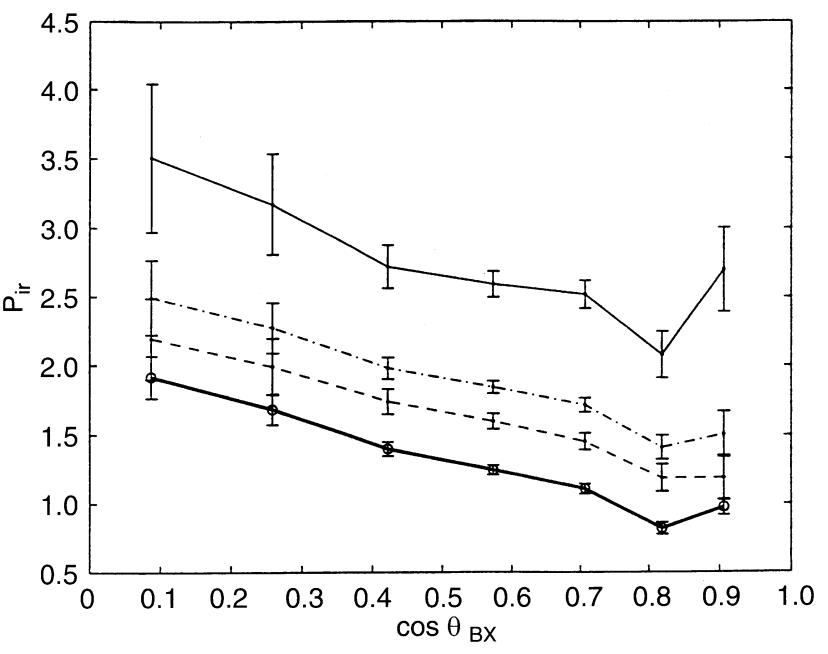

Fig. 6. Dependence of $P_{i r}$ on $\cos \theta_{B X}$ deduced from observations (thick solid line) and the emission models: adiabatic reflection of solarwind ions (solid line), leakage from magnetosheath with magnetic moment conservation (dash-dot line), and leakage along the shock normal (dashed line)

reflection process of solar-wind ions with magnetic moment conservation, the agreement of the results of the calculation with the observational measure is worse than for the two other emission processes (solid line). The difference in speed is more than $1.5 V_{s w}$ and the theoretical value is around twice the measured value. For leakage particles with magnetic moment conservation (dash-dot line), the curves that represent the theoretical values and the observed ones are remarkably parallel with a difference of about $0.6 V_{s w}$. Finally, the leakage of particles along the shock normal (dashed line) produces the smallest deviation between the computed values and the observed values, around $0.3 V_{s w}$. This indicates that the primary source of intermediate ion distributions may be the leakage of magnetosheath particles, an explanation consistent with theoretical work performed by Edmiston et al. (1982).

In all cases the same functional form is computed and observed, with a constant difference in speed between the velocities deduced from the observation and the larger velocities computed for the different emission mechanisms. This clearly indicates that the position of the intermediate ion boundary is related to the upstream emission process at the shock surface. We suggest that the difference in velocity between the values deduced from the observations of the boundary position and the values computed from the different emission processes may be attributed to a wave-particle interaction mechanism. Indeed, theoretical studies of the linear or quasilinear development of the beam-plasma instability show that pitch-angle scattering of beam particles reduces their parallel energy (Winske and Leroy, 1984). These authors also have carried out a simulation showing how diffuse ions are produced in an electromagnetic ionbeam instability. Using both linear and quasi-linear theory, they have computed the spatially averaged momentum and energy exchange rates among the different particle species (beam ions, solar-wind ions 
and electrons). In both cases the ion beam loses momentum to the solar-wind ions.

\section{Conclusion}

We have undertaken a statistical investigation of the location of the intermediate ion population in the Earth's foreshock. Our study reveals the existence of a spatial boundary for intermediate and gyrating ion populations. We have found that for cone angles $\theta_{B X}$ near $45^{\circ}$ the intermediate ion foreshock boundary coincides with the ULF foreshock boundary reported earlier by Greenstadt and Baum (1986) obtained using magnetometer data. This confirms the association of the intermediate (and gyrating) ion distributions with ULF foreshock waves. At smaller cone angles, the two boundaries correspond somewhat less. On the basis of momentum computation, we suggest that this boundary corresponds to the onset of the diffuse ion region. The primary consequence is that fixed growth rate processes associated with instabilities are incompatible with the geometric location of the boundary. The boundary location seems in agreement with an interaction model involving a nonuniform injection of energetic protons from the shock and hydromagnetic waves (Skadron et al., 1988).

To understand the variation of the boundary position with the interplanetary cone angle $\theta_{B X}$, we derived the momentum associated with particles which travel upstream along this boundary, thereby showing that the results are consistent with classical acceleration mechanisms at the shock. Among the four acceleration processes, namely adiabatic refection of solar-wind ions, specular reflection, escape of magnetosheath ions with magnetic moment conservation, and escape of magnetosheath ions along the shock normal, we found that (1) the specular reflection mechanism is excluded, since the boundary intersects the shock with $\theta_{B n}$ larger than $45^{\circ}$, and (2) the magnetosheath ion leakage mechanisms are more suitable for representing the observed behavior. However, a constant difference between the acceleration-model-predicted momentum and the associated particle momentum is found with the model predictions systematically larger than the corresponding observed values. We suggest that this difference may be due to some momentum exchange between the incident solarwind population and the backstreaming particles through the wave-particle interaction resulting from a beam-plasma instability. This momentum loss could provide an observational constraint on modeling the whole process, which leads first to intermediate, and finally to diffuse, ion distributions.

Acknowledgements. K. Meziane thanks R. K. Elsen for help in editing the revision of this paper. Tropical Editor K.-H. Glaßmeier thanks two referees for their help in evaluating this paper.

\section{Appendix}

Derivation of $P_{\text {ir }}$ value associated with the intermediate ion foreshock boundary

The guiding center velocity $\vec{V}_{G C}$ of a backstreaming particle in the foreshock region in the spacecraft frame is the sum of its velocity parallel to magnetic field $\vec{V}_{\|}$and the convection velocity $\vec{V}_{D}$, with $V_{D}=V_{S w} \sin \theta_{B X}$ and $V_{\|}=P_{i B} V_{s w}$, where $\theta_{B X}$ is the cone angle, $V_{s w}$ the solarwind velocity, and $P_{i B}$ is a multiplicative factor. We can also express the guiding center velocity modulus in terms of the solar-wind velocity as $V_{G C}=P_{i r} V_{s w}$. We deduce then

$P_{i r}^{2}=P_{i B}^{2}+\sin ^{2} \theta_{B X}$.

Let $P V_{s w}$ be the particle parallel velocity in the solarwind frame. From Fig. 7, we can write

$\vec{V}_{G C}=P V_{s w} \frac{\vec{B}}{B}+\vec{V}_{s w}$

and

$P=P_{i B}+\cos \theta_{B X}$

Using Eqs. (A1)-(A3), we deduce

$P_{i r}=\left(1+P^{2}-2 P \cos \theta_{B X}\right)^{1 / 2}$.

Let $\alpha$ be the angle between the solar-wind direction ( $X$-axis) and the guiding center velocity vector of a particle which travels along the intermediate ion boundary. From Fig. 7 we can write:

$\operatorname{tg} \alpha=\frac{P \sin \theta_{B X}}{P \cos \theta_{B X}-1}$.

Using Eqs. (A4) and (A5), we can eliminate $P$ to obtain $P_{i r}$ in terms of $\operatorname{tg} \alpha$. This last quantity is the slope of the intermediate ion boundary in the $(X, \eta)$ plane, which is obtained from the observations.

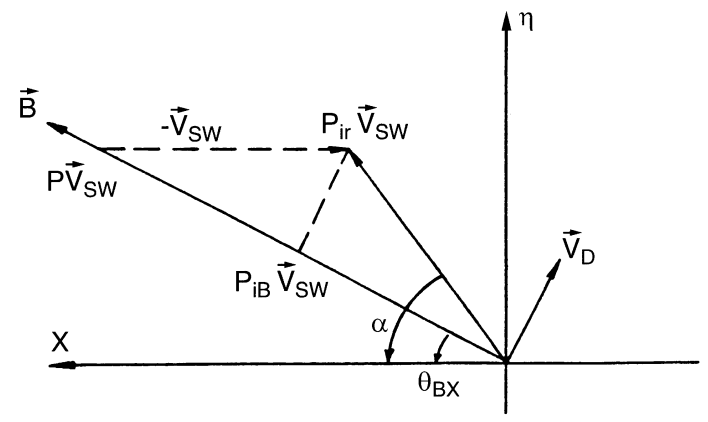

Fig. 7. Schematic illustration of the decomposition of guiding center velocity vector $\vec{V}_{G C}$ of a backstreaming particle in the Earth's foreshock 


\section{References}

Anderson, K. A., R. P. Lin, R. J. Paoli, G. K. Parks, C. S. Lin, H. Rème, J. M. Bosqued, F. Martel, F. Cotin, and A. Cros, An experiment to study energetic particle fluxes in and beyond the Earth's outer magnetosphere, IEEE Trans. Geosci. Elec., GE16, 213, 1978 .

Bonifazi, C., and G. Moreno, Reflected and diffuse ions backstreaming from the Earth's bow shock. 1, Basic properties, J. Geophys. Res., 86, 4397, 1981.

Edmiston, J. P., C. F. Kennel, and D. Eichler, Escape of heated ions upstream of quasi-parallel shocks, Geophys. Res. Lett., 9, 531, 1982

Fuselier, S. A., Suprathermal ions upstream and downstream from the Earth's bow shock, in Solar wind sources of magnetospheric ultra-low frequency, Geophys. Monogr. Ser., 81, Ed. M. Engebretson, M. Takahashi, and M. Scholer, AGU, Washington, D. C., pp. 107-119, 1994.

Fuselier, S. A., Ion distribution in the Earth's foreshock upstream from the bow shock, Adv. Space Res., 15, 8/9, 43, 1995.

Fuselier, S. A., and M. F. Thomsen, $\mathrm{He}^{2+}$ in field-aligned beams: ISEE results, Geophys. Res. Lett., 19, 437, 1992.

Fuselier, S. A., M. F. Thomsen, J. T. Gosling, S. J. Bame, and C. T. Russell, Gyrating and intermediate ion distributions upstream from the Earth's bow shock, J. Geophys. Res., 91, 91,1986.

Gary, S. P., J. T. Gosling, and D. W. Forslund, The electromagnetic ion beam instability upstream of the Earth's bow shock, $J$. Geophys. Res., 86, 6691, 1981.

Gosling, J. T., J. R. Asbridge, S. J. Bame, G. Paschmann, and N. Sckopke, Observations of two distinct populations of bow shock ions in the upstream solar wind, Geophys. Res. Lett., 5, 957, 1978.

Gosling, J. T., M. F. Thomsen, S. J. Bame, W. C. Feldman, G. Paschmann, and N. Sckopke, Evidence for specularly reflected ions upstream from the quasi-parallel bow shock, Geophys. Res. Lett., 9, 1333, 1982.

Greenstadt, E. W., and W. Baum, Earth's compressional foreshock boundary revisited: observations by the ISEE 1 magnetometer, J. Geophys. Res., 91, 9001, 1986.

Gurgiolo, C., G. K. Parks, B. H. Mauk, C. S. Lin, K. A. Anderson, R. P. Lin, and H. Rème, Non $\boldsymbol{E} \times \boldsymbol{B}$ ordered ion beams upstream of the Earth's bow shock, J. Geophys. Res., 86, 4451, 1981.

Gurgiolo, C., G. K. Parks, and B. H. Mauk, Upstream gyrophasebunched ions: a mechanism for creation at the bow shock and the growth of velocity space structure through gyrophase mixing, J. Geophys. Res., 88, 9093, 1983.

Hada, T., C. K. Kennel, and T. Terasawa, Excitation of compressional waves and the formation of shocklets in the Earth's foreshock, J. Geophys. Res., 92, 4423, 1987.

Hoppe, M. M., C. T. Russell, L. A. Frank, T. E. Eastman, and E. W. Greenstadt, Upstream hydromagnetic waves and their association with backstreaming ion populations: ISEE 1 and 2 observations, J. Geophys. Res., 86, 4471, 1981.

Hoshino, M., and T. Teresawa, Numerical study of the upstream waves excitation mechanism, 1. nonlinear phase bunching of beam ions, J. Geophys. Res., 90, 57, 1985.

Ipavich, F. M., J. T. Gosling, and M. Scholer, Correlation between the $\mathrm{He} / \mathrm{H}$ ratio in upstream particle events and in the solar wind, J. Geophys. Res., 89, 1501, 1984.

Ipavich, F. M., G. Gloeckler, D. C. Hamilton, L. M. Kistler, and J. T. Gosling, Protons and alpha particles in field-aligned beams upstream of the bow shock, Geophys. Res. Lett., 15, 1153, 1988.

Le, G., and C. T. Russell, A study of ULF wave foreshock morphology: ULF foreshock boundary, Planet. Space Sci., 40, 1203,1992
Lee, M. A., Coupled hydromagnetic wave excitation and ion acceleration upstream of the Earth's bow shock, J. Geophys. Res., 87, 5063, 1982.

Lee, M. A., and G. Skadron, A simple model for formation of reflected, intermediate and diffuse ion distributions upstream of Earth's bow shock, J. Geophys. Res., 90, 39, 1985.

Leroy, M. M., D. Winske, C. C. Goodrich, C. S. Wu, and K. Papadopoulos, The structure of perpendicular bow shocks, $J$. Geophys. Res., 87, 5081, 1982.

Möbius, E., M. Scholer, N. Sckopke, H. Lühr, G. Paschmann, and D. Hovestadt, The distribution of diffuse ions and the magnetic field power spectrum upstream of the Earth's bow shock, Geophys. Res. Lett., 14, 681, 1987.

Paschmann, G., N. Sckopke, S. J. Bame, J. R. Asbridge, J. T. Gosling, C. T. Russell, and E. W. Greenstadt, Association of low-frequency waves with suprathermal ions in the upstream solar wind, Geophys. Res. Lett., 6, 209, 1979.

Paschmann, G., N. Sckopke, I. Papamastorakis, J. R. Asbridge, S. J. Bame, and J. T. Gosling, Energization of solar wind ions by reflection from the Earth's bow shock, J. Geophys. Res., 85, 4689, 1980.

Paschmann, G., N. Sckopke, I. Papamastorakis, J. R. Asbridge, S. J. Bame, and J. T. Gosling, Characteristics of reflected and diffuse ions upstream from the Earth's bow shock, J. Geophys. Res., 86, 4355, 1981.

Potter, D. W., High time resolution characteristics of intermediate ion distributions upstream of the Earth's bow shock, J. Geophys. Res., 90, 261, 1985.

Quest, K. B., Theory and simulation of collisionless parallel shocks, J. Geophys. Res., 93, 9649, 1988.

Sanderson, J. J., and R. A. Uhrig Jr, Extended Rankine-Hugoniot relations for collisionless shocks, J. Geophys. Res., 83, 1395, 1978.

Scholer, M., Diffuse ions at quasi-parallel collisionless shock: simulations, Geophys. Res. Lett., 17, 1821, 1990.

Scholer, M., and T. Teresawa, Ion reflection and dissipation at quasi-parallel collisionless shocks, Geophys. Res. Lett., 17, 119, 1990.

Schwartz, S. J., M. F. Thomsen, and J. T. Gosling, Ions upstream of the Earth's bow shock: a technical comparison of alternative source populations, J. Geophys. Res., 88, 2039, 1983.

Sentman, D., J. P. Edmiston, and L. A. Frank, Instabilities of lowfrequency parallel propagation electromagnetic waves in the Earth's foreshock region, J. Geophys. Res., 86, 4365, 1981.

Skadron, G., R. D. Holdaway, and M. A. Lee, Formation of the wave boundary in the Earth's foreshock, J. Geophys. Res., 93, 11354, 1988.

Thomsen, M. F., J. T. Gosling, S. J. Bame, W. C. Feldman, G. Paschmann, and N. Sckopke, Field-aligned ion beams upstream of the Earth's bow shock: evidence for a magnetosheath source, Geophys. Res. Lett., 10, 1207, 1983a.

Thomsen, M. F., S. J. Schwartz, and J. T. Gosling, Observational evidence on the origin of ions upstream of the Earth's bow shock, J. Geophys. Res., 88, 7843, 1983b.

Thomsen, M. F., J. T. Gosling, S. J. Bame, and C. T. Russell, Gyrating ions and large-amplitude monochromatic MHD waves upstream of the Earth's bow shock, J. Geophys. Res., 90, 267, 1985.

Trattner, K. J., E. Möbius, M. Scholer, B. Klecker, M. Hilchenbach, H. Lühr, Statistical analysis of diffuse ion events upstream of the bow shock, J. Geophys. Res., 99, 13389, 1994.

d'Uston, C., K. Meziane, and A. Saint-Marc, Some aspects of the diffusion in the upstream region of the Earth deduced from ISEE 1 observations, Adv. Space Res., 6, 71, 1986.

Winske, D., and M. M. Leroy, Diffuse ions produced by electromagnetic ion beam instability, J. Geophys. Res., 89, 2673, 1984. 